\title{
Adrenal Metastasis and Hemorrhage Secondary to Hepatocellular Carcinoma
}

\author{
Shintaro Akiyama, Tsunao Imamura, Rikako Koyama, \\ Tetsuo Tamura, Yuko Koizumi and Kazuo Takeuchi
}

\begin{abstract}
A 66-year-old man was admitted to undergo treatment for the sudden onset of hypogastric pain radiating to the left flank. Based on the clinical presentation and radiological findings, left adrenal hemorrhage secondary to hepatocellular carcinoma was diagnosed. Although the patient was hemodynamically stable, anemia progressed over the first four days after admission and a blood transfusion was subsequently initiated, which was effective. According to the results of interval imaging with computed tomography, elective transcatheter embolization (TAE) was performed nine days after admission to treat the bleeding from the adrenal tumor. After TAE, the patient's abdominal pain resolved, with no further progression of anemia.
\end{abstract}

Key words: hepatocellular carcinoma, adrenal metastasis, retroperitoneal hemorrhage

(Intern Med 54: 1513-1517, 2015)

(DOI: 10.2169/internalmedicine.54.4115)

\section{Introduction}

The adrenal gland is a common site of metastasis of hepatocellular carcinoma (HCC). The incidence of adrenal metastasis is approximately $10 \%(1,2)$, and adrenal hemorrhage due to metastatic $\mathrm{HCC}$ has been reported in a few cases $(3,4)$. However, there are currently no definitive guidelines for the treatment of adrenal hemorrhage resulting from metastasis, and transcatheter arterial embolization (TAE) and/or surgical intervention tend to be applied to stop tumor bleeding. We herein report a case of adrenal hemorrhage secondary to HCC metastasis. After referring to the results of interval imaging with computed tomography (CT) and blood tests, we successfully treated the adrenal bleeding with elective TAE. In severely cirrhotic patients with adrenal hemorrhage due to HCC who are hemodynamically stable, interval imaging to determine the possibility of resolution of the hematoma prior to elective TAE is thought to be a more feasible treatment strategy than performing surgery in these high-risk cases.

\section{Case Report}

A 66-year-old man presented to the emergency department of another hospital with the sudden onset of hypogastric pain radiating to the left flank and was ultimately referred to our hospital for further evaluations. The patient's past medical history included the identification of the antibody to hepatitis $\mathrm{C}$ virus on a routine medical checkup at 57 years of age. HCC was subsequently detected on abdominal ultrasonography (US) two years later. Although transcatheter arterial chemoembolization (TACE) was performed a total of six times, the HCC continued to grow and began to invade the portal vein. Therefore, radiation therapy was initiated, with a total of 60 gray (Gy) administered to the lesion. CT performed four months before admission showed a solitary mass in the left adrenal gland measuring $25 \times 20 \mathrm{~mm}$ (Fig. 1A); two months later (two months prior to the current admission) the tumor had increased in size to $50 \times 30 \mathrm{~mm}$ (Fig. 1B).

On a physical examination, the patient was afebrile, with a regular pulse of 83 beats/min and blood pressure of 143/ $85 \mathrm{mmHg}$, and there was tenderness in the left upper quadrant. The laboratory findings included the following data

Department of Gastroenterology, Toranomon Hospital, Japan

Received for publication September 15, 2014; Accepted for publication October 26, 2014

Correspondence to Dr. Shintaro Akiyama, akiyama_s@hotmail.co.jp 

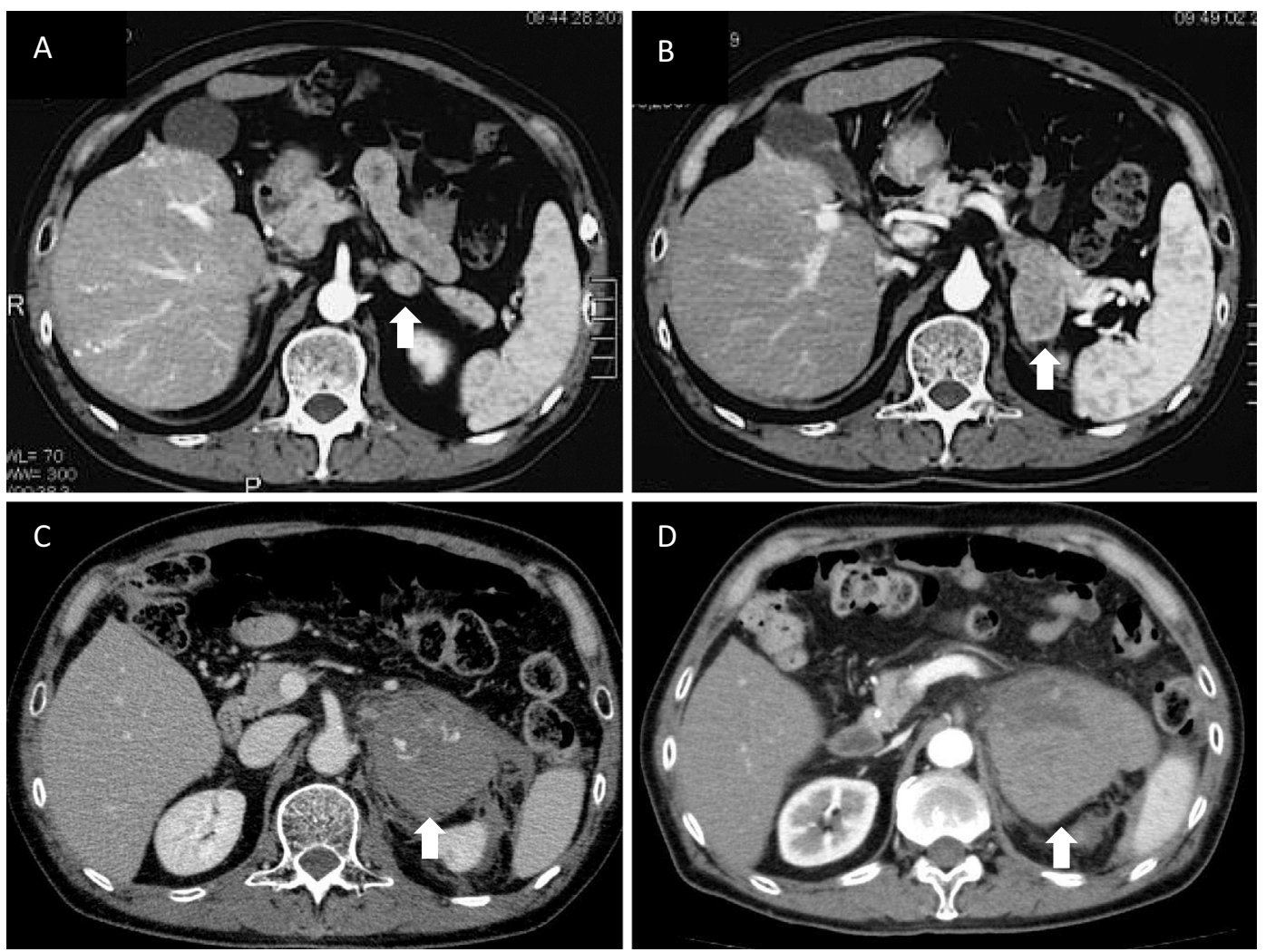

Figure 1. A, B: Computed tomography (CT) scan obtained at four months (A) and two months (B) before admission revealed a left adrenal contrast-enhanced mass measuring $25 \times 20 \mathrm{~mm}$ and $50 \times 30$ mm, respectively (white arrows). C, D: Findings of interval imaging on CT. Contrast-enhanced CT performed on admission showed a $72 \times 62 \mathrm{~mm}$ contrast-enhanced mass in the left adrenal gland with surrounding hematoma formation in the left perirenal fascia and splenic hilum extending to the left subdiaphragmatic space (C, white arrow). A CT scan obtained four days (D) after admission showed growth of the left adrenal mass (white arrow).

(Table): albumin=3.1 g/dL; total bilirubin=2.2 mg/dL; hemoglobin=9.3 $\mathrm{g} / \mathrm{dL} ; \alpha$-fetoprotein $=17 \mu \mathrm{g} / \mathrm{dL}$ (reference range: 0-20 $\mu \mathrm{g} / \mathrm{dL}$ ); des-gamma carboxyprothrombin $(\mathrm{DCP})=364$ $\mathrm{U} / \mathrm{mL}$ (reference range: $0-30 \mathrm{U} / \mathrm{mL}$ ); and prothrombin time international normalized ratio $(\mathrm{PT}$ INR $)=1.12$. The Child Pugh classification was class B. An endocrine survey revealed a cortisol level of $22.0 \mu \mathrm{g} / \mathrm{dL}$ (reference range: 4.5 $21.1 \mu \mathrm{g} / \mathrm{dL}$ ), adrenocorticotropic hormone (ACTH) level of $64.1 \mathrm{pg} / \mathrm{mL}$ (reference range: 7.2-63.3 pg/mL), adrenaline level of $0.02 \mathrm{ng} / \mathrm{mL}$ (reference range: $\leq 0.1 \mathrm{ng} / \mathrm{mL}$ ), noradrenaline level of $0.10 \mathrm{ng} / \mathrm{mL}$ (reference range: $0.10-0.45 \mathrm{ng} /$ $\mathrm{mL}$ ) and dopamine level of $0.01 \mathrm{ng} / \mathrm{mL}$ (reference range: $0.02 \mathrm{ng} / \mathrm{mL}$ ). Contrast-enhanced CT on admission revealed a $72 \times 62 \mathrm{~mm}$ contrast-enhanced mass in the left adrenal gland with surrounding hematoma formation in the left perirenal fascia and splenic hilum extending to the left subdiaphragmatic space (Fig. 1C). There also appeared to be extravasation from the solitary mass, and we strongly suspected adrenal hemorrhage as a result of adrenal metastasis of HCC. On hospital day 4, although the patient's vital signs remained stable, the hemoglobin level decreased from $10.9 \mathrm{~g} /$ $\mathrm{dL}$ to $7.6 \mathrm{~g} / \mathrm{dL}$; therefore, 4 units of packed red blood cells were administered. The blood transfusion was effective for treating the anemia, and a repeat $\mathrm{CT}$ scan revealed that the left adrenal hematoma had become enlarged (Fig. 1D). Hence, TAE was performed on hospital day 9 to treat the adrenal hemorrhage.

During TAE, transfemoral abdominal angiography identified the superior adrenal artery, a branch of the inferior phrenic artery, as the site of bleeding (Fig. 2A). Embolization was performed using Gelpart (gelatin particles) and Lipiodol (ethiodized oil), and the adrenal bleeding was controlled successfully (Fig. 2B). In addition, the patient's abdominal pain completely resolved, and he was discharged 19 days after admission. Following the embolization procedure, no further progression in anemia was noted during the follow-up period since the time of discharge to the present.

\section{Discussion}

Adrenal hemorrhage secondary to HCC metastasis is very rare. We reviewed the English medical literature (using the key words "adrenal hemorrhage, hepatocellular carcinoma") and found only two case reports of adrenal hemorrhage secondary to HCC metastasis $(3,4)$.

In a review of 133 reported cases of hemorrhagic adrenal masses, the most commonly reported pathologic diagnosis was pheochromocytoma (64 cases, $48 \%$ ), followed by me- 
Table. Laboratory Data on Admission.

\begin{tabular}{lrlllll}
\hline Complete Blood Counts & & & Biochemistry & \\
WBC & 5,000 & $/ \mu \mathrm{L}$ & TP & 7.6 & $\mathrm{~g} / \mathrm{dL}$ \\
$\mathrm{RBC}$ & $2.62 \times 10^{6}$ & $/ \mu \mathrm{L}$ & Alb & 3.1 & $\mathrm{~g} / \mathrm{dL}$ \\
$\mathrm{Hb}$ & 9.3 & $\mathrm{~g} / \mathrm{dL}$ & $\mathrm{T}-\mathrm{Bil}$ & 2.2 & $\mathrm{mg} / \mathrm{dL}$ \\
$\mathrm{Ht}$ & 25.9 & $\%$ & AST & 119 & $\mathrm{U} / \mathrm{L}$ \\
PLT & $8.8 \times 10^{4}$ & $/ \mu \mathrm{L}$ & ALT & 99 & $\mathrm{U} / \mathrm{L}$ \\
& & & $\mathrm{LDH}$ & 337 & $\mathrm{U} / \mathrm{L}$ \\
Coagulation & & & ALP & 342 & $\mathrm{U} / \mathrm{L}$ \\
APTT & 26.3 & $\mathrm{sec}$ & Y-GTP & 333 & $\mathrm{U} / \mathrm{L}$ \\
PT & 77.2 & $\%$ & BUN & 19 & $\mathrm{mg} / \mathrm{dL}$ \\
PT-INR & 1.12 & & Cr & 0.76 & $\mathrm{mg} / \mathrm{dL}$ \\
Serology & & & Hormone Analysis & & \\
CRP & 9.1 & $\mathrm{mg} / \mathrm{dL}$ & Cortisol & 22.0 & $\mu \mathrm{gg} / \mathrm{dL}$ \\
& & & ACTH & 64.1 & $\mathrm{pg} / \mathrm{mL}$ \\
Tumor markers & & & Adrenaline & 0.02 & $\mathrm{ng} / \mathrm{mL}$ \\
AFP & 17 & $\mu \mathrm{g} / \mathrm{L}$ & Noradrenaline & 0.10 & $\mathrm{ng} / \mathrm{mL}$ \\
DCP & 364 & $\mathrm{U} / \mathrm{mL}$ & Dopamine & 0.01 & $\mathrm{ng} / \mathrm{mL}$ \\
\hline
\end{tabular}

WBC: White Blood Cells, RBC: Red Blood Cells, Hb: Hemoglobin, Ht: Hematocrit, PLT: Platelets, TP: Total Protein, Alb: Albumin, T-Bil: Total Bilirubin, AST: Aspartate Transaminase, ALT: Alanine Aminotransferase, LDH: Lactate Dehydrogenase, ALP: Alkaline Phosphatase, $\mathrm{Y}^{-G T P}$ : $\mathrm{Y}^{-G l u t a m y l}$ Transpeptidase, BUN: Blood Urea Nitrogen, Cr: Creatinine, APTT: Activated Partial Thromboplastin Time, PT: Prothrombin Time, INR: International Normalized Ratio, AFP: $\alpha$-Fetoprotein, DCP: Des-Gamma Carboxyprothrombin, CRP: C-Reactive Protein, ACTH: Adrenocorticotropic Hormone

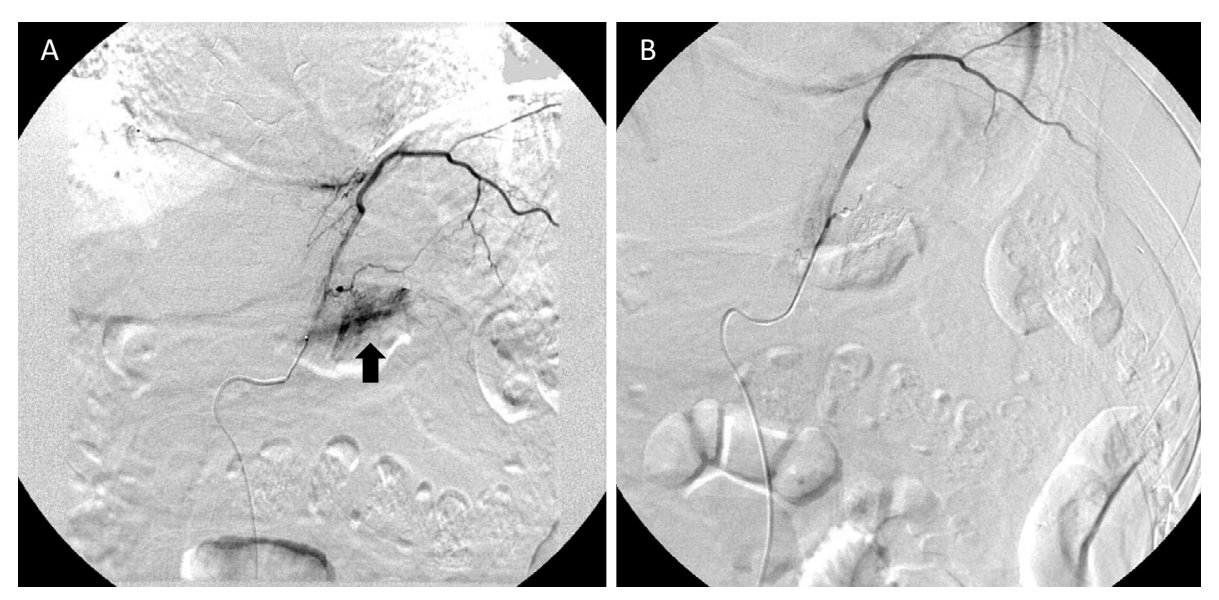

Figure 2. Findings of transcatheter embolization (TAE). A: Transfemoral abdominal angiography identified the superior adrenal artery, a branch of the inferior phrenic artery, as the site of bleeding (black arrow). B: Embolization was performed using Gelpart (gelatin particles) and Lipiodol (ethiodized oil); the adrenal bleeding was successfully controlled.

tastatic lesions to the adrenal gland (27, 20\%), pseudocysts/ adenoma $(23,17 \%)$, myelolipoma $(13,10 \%)$, pseudocysts during pregnancy $(5,4 \%)$ and lipoma $(1,1 \%)$. The most common malignant lesions were adrenocortical tumors (9 cases, $33 \%$ ), followed by metastasis to the lungs (9,33\%), kidneys $(2,7 \%)$ and one case $(4 \%)$ each of the following cancers: colon, thyroid, bladder, soft tissue, bone, hepatic angiosarcoma and hepatoma. Most patients (105 cases, 79\%) underwent adrenalectomy alone, seven (5\%) underwent TAE prior to adrenalectomy, two (2\%) underwent embolization alone and $19(14 \%)$ did not undergo embolization or adrenalectomy (5). Marti et al. established a treatment algorithm for patients with hemorrhagic adrenal masses. According to this algorithm, if patients with nonfunctional hemor- rhagic adrenal tumors are hemodynamically stable, interval imaging is recommended to assess for possible resolution of the hematoma. On the other hand, in patients who are hemodynamically unstable, TAE prior to emergent laparotomy is recommended. In the current case, interval imaging was performed prior to TAE because the patient was hemodynamically stable, and the elevated ACTH and cortisol levels and normal catecholamine level did not indicate a hormonally active tumor, but rather physiological stress. We suspected that the adrenal hemorrhage had resolved naturally because the patient's vital signs were stable and the hematoma was localized in the left retroperitoneal space. Indeed, Marti J.L. et al. reported a case of sepsis due to a perforated colon in which the left adrenal hematoma resolved after six 
months as well as a case of adrenal metastasis of lung cancer in which the hematoma decreased in size six months after chemotherapy (5). In the current case, because interval imaging revealed that the hematoma had progressively become enlarged and the anemia was exacerbated simultaneously, TAE was chosen to treat the adrenal hemorrhage. Considering the outcome in this case, conducting interval imaging and blood tests prior to elective TAE should be a strategy for managing adrenal hemorrhage secondary to HCC metastasis, similar to the management strategies for intrahepatic HCC rupture. HCC with adrenal metastasis is accompanied by advanced liver damage, placing these patients at high risk for surgery. We believe the approach taken in the current case is more feasible for treating liver cirrhosis patients.

In general, ruptured $\mathrm{HCC}$ occurs in $3 \%$ to $15 \%$ of patients with HCC (6). Although the exact mechanisms of HCC rupture remain unknown, it has been hypothesized that HCC rupture arises from rapid tumor growth and necrosis, resulting in splitting of the overlying nontumorous liver parenchyma and/or vessel erosion, with increased intratumoral pressure associated with occlusion of the hepatic veins due to coagulopathy, vascular dysfunction and tumor thrombosis or invasion (6). The precise mechanisms of adrenal hemorrhage caused by HCC metastasis are also unclear due to the rarity of this condition. The adrenal gland has a rich blood supply and is particularly susceptible to hemorrhage (7). An enhanced blood supply or disturbance of blood efflux may easily induce vascular splitting and thrombosis. We hypothesized that the rapid growth of HCC enhances the adrenal blood supply and induces the formation of vascular splits. Indeed, rapid enlargement of the tumor (from $25 \times 20 \mathrm{~mm}$ to $50 \times 30 \mathrm{~mm}$ over two months) was detected prior to admission in the current case (Fig. 1A, B). The risk of adrenal hemorrhage due to HCC metastasis is associated with the speed of tumor growth.

The adrenal gland is the fourth most frequent site of distant metastasis of HCC after the lungs, lymph nodes and bone $(1,2)$. The management of adrenal metastasis remains controversial. In a previous report, the median survival duration was only 11.05 months in 30 patients with adrenal metastases. Among the 25 patients with well-controlled intrahepatic lesions, the median survival time was 21.41, 11.05 and 5.64 months for the patients who received adrenalectomy, non-surgical therapies (including TACE or chemotherapy and radiotherapy) and no treatment, respectively (8). On the other hand, if intrahepatic HCC is not well controlled or extrahepatic HCC, including adrenal metastasis, is detected, radiofrequency ablation (RFA) with and without TAE $(9,10)$, percutaneous ethanol injection (PEIT) (11), radiation therapy (12) and/or systemic chemotherapy (13) are applicable. To our knowledge, there are no previous studies comparing these treatment modalities.

In the current case, surgical resection of adrenal metastasis of HCC was not indicated before or after the onset of adrenal bleeding because the intrahepatic HCC was not well controlled, as evidenced by portal invasion. Although RFA with or without TACE or PEIT would have been applicable for controlling the adrenal metastasis, we did not select these methods because they are not well established. Furthermore, external beam radiation for the left adrenal lesion carried the risk of irradiating the intestines, and TACE monotherapy is thought to be ineffective in such cases because the adrenal gland has three feeder arteries (inferior phrenic artery, abdominal aorta, renal artery). Hence, we judged the only treatment alternative to be careful observation of the adrenal metastasis of HCC prior to the development of bleeding. However, adrenal bleeding occurred unexpectedly, and treatment with systemic chemotherapy and TACE was planned to manage the left adrenal HCC metastasis.

In conclusion, we treated a case of adrenal metastasis and hemorrhage secondary to HCC. Similar to that observed in cases of intrahepatic HCC rupture, if the patient is hemodynamically stable, interval imaging to assess for possible resolution of the hematoma prior to performing elective TAE is considered a useful strategy for management in cases of adrenal hemorrhage secondary to HCC metastasis, as cirrhotic patients tend to have advanced liver damage and are therefore at high risk for surgery.

\section{The authors state that they have no Conflict of Interest (COI).}

\section{References}

1. Natsuizaka M, Omura T, Akaike T, et al. Clinical features of hepatocellular carcinoma with extrahepatic metastases. J Gastroenterol Hepatol 20: 1781-1787, 2005.

2. Katyal S, Oliver JH 3rd, Peterson MS, Ferris JV, Carr BS, Baron RL. Extrahepatic metastases of hepatocellular carcinoma. Radiology 216: 698-703, 2000.

3. Vasinanukorn P, Rerknimitr R, Sriussadaporn S, et al. Adrenal hemorrhage as the first presentation of hepatocellular carcinoma. Intern Med 46: 1779-1782, 2007.

4. Yang PW, Wang WY, Yang CH, Chou CC, Yen DH, Chou J. Treatment of massive retroperitoneal hemorrhage from adrenal metastasis of hepatoma. J Chin Med Assoc 70: 126-131, 2007.

5. Marti JL, Millet J, Sosa JA, Roman SA, Carling T, Udelsman R. Spontaneous adrenal hemorrhage with associated masses: etiology and management in 6 cases and a review of 133 reported cases. World J Surg 36: 75-82, 2012.

6. Lai EC, Lau WY. Spontaneous rupture of hepatocellular carcinoma. Arch Surg 141: 191-198, 2006.

7. Simon DR, Palese MA. Clinical update on the management of adrenal hemorrhage. Curr Urol Rep 10: 78-83, 2009.

8. Park JS, Yoon DS, Kim KS, et al. What is the best treatment modality for adrenal metastasis from hepatocellular carcinoma? J Surg Oncol 96: 32-36, 2007.

9. Yamakado K, Anai H, Takaki H, et al. Adrenal metastasis from hepatocellular carcinoma: radiofrequency ablation combined with adrenal chemoembolization in six patients. AJR Am J Roentgenol 192: W300-W305, 2009.

10. Kuehl H, Stattaus J, Forsting M, Antoch G. Transhepatic CTguided radiofrequency ablation of adrenal metastases from hepatocellular carcinoma. Cardiovasc Intervent Radiol 31: 1210-1214, 2008.

11. Shibata T, Maetani Y, Ametani F, Itho K, Konishi J. Percutaneous 
Intern Med 54: 1513-1517, 2015 DOI: 10.2169/internalmedicine.54.4115

ethanol injection for treatment of adrenal metastasis from hepatocellular carcinoma. AJR Am J Roentgenol 174: 333-335, 2000.

12. Zeng ZC, Tang ZY, Fan J, et al. Radiation therapy for adrenal gland metastasis from hepatocellular carcinoma. Jpn J Clin Oncol
35: 61-67, 2005.

13. Aramaki M, Kawano K, Kai T, et al. Treatment for extrahepatic metastasis of hepatocellular carcinoma following successful hepatic resection. Hepatogastroenterology 46: 2931-2934, 1999.

(C) 2015 The Japanese Society of Internal Medicine http://www.naika.or.jp/imonline/index.html 